\title{
Global dynamics of humoral immunity Chikungunya virus with two routes of infection and Holling type-II
}

\author{
A. M. Elaiw ${ }^{a, *}$, S. E. Almalkib ${ }^{b}$ A. D. Hobiny ${ }^{a}$ \\ ${ }^{a}$ Department of Mathematics, Faculty of Science, King Abdulaziz University, P. O. Box 80203, Jeddah 21589, Saudi Arabia. \\ b Jeddah College of Technology, Technical and Vocational Training Corporation, P. O. Box 17608, Jeddah 21494, Saudi Arabia.
}

\begin{abstract}
In this work, we analyze the global dynamics of within-host Chikungunya virus (CHIKV) infection model with humoral immune response. We incorporate two modes of infections, attaching a CHIKV to a host monocyte, and contacting an infected monocyte with an uninfected monocyte. The infection incident rate is given by Holling type-II. The basic reproduction number $\mathcal{R}_{0}$ is used to prove that the CHIKV-free equilibrium $E_{0}$ is globally asymptotically stable when $\mathcal{R}_{0} \leqslant 1$ and the infected equilibrium $E_{1}$ is globally asymptotically stable when $\mathcal{R}_{0}>1$. Numerical simulations have been performed to confirm the theoretical results.
\end{abstract}

Keywords: Chikungunya virus, holling type-II, global stability, Lyapunov function, viral and cellular infections.

2010 MSC: 34D23, 93D20, 93D05, 93C55.

(C)2019 All rights reserved.

\section{Introduction}

Mosquito is one of the dangerous insect throughout the world. It can carry and spread viruses to humans and animals causes many of deaths every year. A great efforts has been paid to develop and analyze mathematical models that describe the population dynamics of mosquito-borne diseases such as Zika $[2,4,7]$, dengue $[1,27,43,48]$, malaria [3, 5, 6, 36], yellow fever [40] and chikungunya [8-10, 34, 3739, 46]. Chikungunya virus (CHIKV) is transmitted to humans by infected Aedes albopictus and Aedes agypti mosquito. CHIKV causes severe joint and muscle pain, fever, rash, headache, nausea and fatigue. Wang and Liu [45] have proposed and studied a within-host CHIKV dynamics model which contains four compartments, uninfected-monocytes (s), infected monocytes (y), free CHIKV particles ( $p$ ) and antibodies $(x)$. The model has been extended in $[13,14]$ by considering general CHIKV-monocyte incidence rate. In $[13,14,45]$ it has been assumed that the uninfected monocyte becomes infected by contacting with CHIKV (CHIKV-to-monocyte transmission). Long and Heise [35] have reported that the CHIKV can also spread by infected-to-monocyte transmission. Mathematical models of different viruses with both cellular and

\footnotetext{
*Corresponding author

Email addresses: a_m_elaiw@yahoo.com (A. M. Elaiw), samialmalki0@gmail.com (S. E. Almalki)

doi: $10.22436 /$ jmcs.019.02.01
}

Received: 2016-04-13 Revised: 2019-03-31 Accepted: 2019-04-04 
viral infections have been studied in several works [24, 25, 31-33, 41, 44, 47]. In a very recent work, Elaiw et al. [15] have studied the dynamics of CHIKV model with two routes of infection, however, they did not consider the holling-II. The aim of the present paper is to propose and analyze a CHIKV dynamics model where the infection rate is given by Holling type-II incidence. The proposed model is given as:

$$
\begin{aligned}
& \dot{s}(t)=\beta-\delta s(t)-\frac{\eta_{1} s(t) p(t)}{1+\omega s(t)}-\frac{\eta_{2} s(t) y(t)}{1+\omega s(t)}, \\
& \dot{y}(t)=\frac{\eta_{1} s(t) p(t)}{1+\omega s(t)}+\frac{\eta_{2} s(t) y(t)}{1+\omega s(t)}-\epsilon y(t), \\
& \dot{p}(t)=\pi y(t)-c p(t)-r x(t) p(t), \\
& \dot{x}(t)=\lambda+\rho x(t) p(t)-m x(t) .
\end{aligned}
$$

The uninfected monocytes are generated monocytes by rate $\beta$, die with rate $\delta s(t)$ and be infected by CHIKV and infected monocytes with rate $\frac{\eta_{1} s(t) p(t)}{1+\omega s(t)}+\frac{\eta_{2} s(t) y(t)}{1+\omega s(t)}$, where $\omega$ is the uninfected monocyte Holling type-II constant, and $\eta_{1}$, and $\eta_{2}$ are the incidence rate constants. Constants $\epsilon, c$, and $m$ represent, respectively, the death rate constants of the infected monocytes, $\mathrm{CHIKV}$, and antibodies. Constant $\pi$ is the production rate constant of the CHIKV from infected monocytes. Antibodies attack the CHIKV at rate $r \chi(t) p(t)$. Once antigen is encountered, the antibodies expand at a constant rate $\lambda$ and proliferate at rate $\rho x(t) p(t)$. All the parameters of the model are positive.

\subsection{Basic properties} (1.4).

The following lemma establishes the nonnegativity and boundedness of the solutions of system (1.1)-

Lemma 1.1. There exist $M_{1}, M_{2}, M_{3}>0$, such that the following compact set is positively invariant for system (1.1)-(1.4);

$$
\Gamma=\left\{(s, y, p, x) \in \mathbb{R}_{\geqslant 0}^{4}: 0 \leqslant s, y \leqslant M_{1}, 0 \leqslant p \leqslant M_{2}, 0 \leqslant x \leqslant M_{3}\right\}
$$

Proof. We have

$$
\begin{aligned}
& \left.\dot{s}\right|_{s=0}=\beta>0, \\
& \left.\dot{y}\right|_{y=0}=\frac{\eta_{1} s p}{1+\omega s} \geqslant 0 \text { for all } s, p \geqslant 0, \\
& \left.\dot{p}\right|_{p=0}=\pi y \geqslant 0 \text { for all } y \geqslant 0, \\
& \left.\dot{x}\right|_{x=0}=\lambda>0 .
\end{aligned}
$$

This shows that $(s(t), y(t), p(t), x(t)) \in \mathbb{R}_{\geqslant 0}^{4}$ with $(s(0), y(0), p(0), x(0)) \in \mathbb{R}_{\geqslant 0}^{4}$. Let us define

$$
\mathrm{H}_{1}(\mathrm{t})=\mathrm{s}(\mathrm{t})+\mathrm{y}(\mathrm{t}), \quad \mathrm{H}_{2}(\mathrm{t})=\mathrm{p}(\mathrm{t})+\frac{\mathrm{r}}{\rho} \mathrm{\rho}(\mathrm{t}) .
$$

Then from Eqs. (1.1)-(1.4) we get

$$
\dot{\mathrm{H}}_{1}(\mathrm{t})=\beta-\delta \mathrm{s}(\mathrm{t})-\epsilon \mathrm{y}(\mathrm{t}) \leqslant \beta-\sigma_{1}(\mathrm{~s}(\mathrm{t})+\mathrm{y}(\mathrm{t}))=\beta-\sigma_{1} \mathrm{H}_{1}(\mathrm{t}),
$$

where, $\sigma_{1}=\min \{\delta, \epsilon\}$. Hence $H_{1}(t) \leqslant M_{1}$, if $H_{1}(0) \leqslant M_{1}$, where $M_{1}=\frac{\beta}{\sigma_{1}}$. It follows that $0 \leqslant s(t), y(t) \leqslant$ $M_{1}$ if $0 \leqslant s(0)+y(0) \leqslant M_{1}$. Moreover, we have

$$
\dot{\mathrm{H}}_{2}(\mathrm{t})=\pi \mathrm{y}(\mathrm{t})-\mathrm{cp}(\mathrm{t})+\frac{\mathrm{r}}{\rho} \lambda-\frac{\mathrm{mr}}{\rho} \chi(\mathrm{t}) \leqslant \pi \mathrm{M}_{1}+\frac{\mathrm{r}}{\rho} \lambda-\sigma_{2}\left(p(t)+\frac{\mathrm{r}}{\rho} \chi(\mathrm{t})\right)=\pi \mathrm{M}_{1}+\frac{\mathrm{r}}{\rho} \lambda-\sigma_{2} \mathrm{H}_{2}(\mathrm{t}),
$$

where, $\sigma_{2}=\min \{c, m\}$. Hence $H_{2}(t) \leqslant M_{2}$, if $H_{2}(0) \leqslant M_{2}$, where $M_{2}=\frac{\pi M_{1}+\frac{r}{\rho} \lambda}{\sigma_{2}}$. Since $p(t)$ and $x(t)$ are all non-negative, then $0 \leqslant p(t) \leqslant M_{2}$ and $0<x(t) \leqslant M_{3}$ if $0<p(0)+\frac{r}{\rho} x(0) \leqslant M_{2}$, where $M_{3}=\frac{\rho M_{2}}{r}$. 


\subsection{Equilibria}

We define the basic reproduction number as:

$$
\mathcal{R}_{0}=\frac{\left(\eta_{1} \pi m+\eta_{2} c m+\eta_{2} r \lambda\right) \beta}{\epsilon(c m+r \lambda)(\delta+\beta \omega)} .
$$

Lemma 1.2. Consider system (1.1)-(1.4), then

- if $\mathcal{R}_{0} \leqslant 1$, then there exists only one equilibrium $\mathrm{E}_{0} \in \Gamma$, and

- if $\mathcal{R}_{0}>1$, then there exist two equilibria $\mathrm{E}_{0} \in \Gamma$ and $\mathrm{E}_{1} \in \stackrel{\circ}{\Gamma}$, where $\stackrel{\circ}{\Gamma}$ is the interior of $\Gamma$.

Proof. Let $\mathrm{E}(s, y, p, x)$ be any equilibrium satisfying

$$
\begin{aligned}
& 0=\beta-\delta s-\frac{\eta_{1} s p}{1+\omega s}-\frac{\eta_{2} s y}{1+\omega s}, \\
& 0=\frac{\eta_{1} s p}{1+\omega s}+\frac{\eta_{2} s y}{1+\omega s}-\epsilon y, \\
& 0=\pi y-c p-r x p \\
& 0=\lambda+\rho x p-m x .
\end{aligned}
$$

By solving Eqs. (1.5)-(1.8) we get two equilibria a CHIKV-free equilibrium $E_{0}=\left(s_{0}, 0,0, x_{0}\right)$, where $s_{0}=\frac{\beta}{\delta}$ and $x_{0}=\frac{\lambda}{\mathrm{m}}$. Moreover, we have

$$
\frac{C_{1} p^{3}+C_{2} p^{2}+C_{3} p+C_{4}}{\bar{C}_{1} p+\bar{C}_{2}}=0
$$

where

$$
\begin{aligned}
& \mathrm{C}_{1}=c \epsilon \rho^{2}\left(-\pi \eta_{1}-c \eta_{2}+c \epsilon \omega\right), \\
& \mathrm{C}_{2}=\mathrm{C}_{21}+\mathrm{C}_{22}+\mathrm{C}_{23}+\mathrm{C}_{24}+\mathrm{C}_{25}, \\
& {\overline{C_{3}}}_{3}=\mathrm{C}_{31}+\mathrm{C}_{32}+\mathrm{C}_{33}+\mathrm{C}_{34}+\mathrm{C}_{35}+\mathrm{C}_{36}, \\
& \mathrm{C}_{4}=\mathrm{m} \pi\left(\mathrm{m} \pi \beta \eta_{1}-\mathrm{cm}\left(\delta \epsilon-\beta \eta_{2}+\beta \epsilon \omega\right)-r \lambda\left(\delta \epsilon-\beta \eta_{2}+\beta \epsilon \omega\right)\right), \\
& \overline{\mathrm{C}_{1}}=\overline{-}+\overline{C_{11}}+ \\
& \overline{\mathrm{C}_{2}}=\overline{\mathrm{C}_{21}}+\overline{\mathrm{C}_{22},}
\end{aligned}
$$

and

$$
\begin{array}{lll}
C_{21}=\rho \pi \eta_{1}(r \epsilon \lambda+\pi \beta \rho), & C_{22}=2 \rho c^{2} m \epsilon\left(\eta_{2}-\epsilon \omega\right), & C_{23}=2 \rho c m \pi \epsilon \eta_{1}, \\
C_{24}=2 \rho c r \epsilon \lambda\left(\eta_{2}-\epsilon \omega\right), & C_{25}=-\pi \rho^{2} c\left(\delta \epsilon-\beta \eta_{2}+\beta \epsilon \omega\right), & C_{31}=-m \pi \eta_{1}(r \epsilon \lambda+2 \pi \beta \rho), \\
C_{32}=c^{2} m^{2} \epsilon\left(-\eta_{2}+\epsilon \omega\right), & C_{33}=r^{2} \lambda^{2} \epsilon\left(-\eta_{2}+\epsilon \omega\right), & C_{34}=r \lambda \pi \rho\left(\delta \epsilon-\beta \eta_{2}+\beta \epsilon \omega\right), \\
C_{35}=c m\left(-m \pi \epsilon \eta_{1}+2 r \epsilon \lambda\left(-\eta_{2}+\epsilon \omega\right)\right), & C_{36}=2 c m \pi \rho\left(\delta \epsilon-\beta \eta_{2}+\beta \epsilon \omega\right), & C_{11}^{-}=\pi \rho r \lambda\left(\eta_{2}-\epsilon \omega\right), \\
C_{12}^{-}=2 \pi \rho m\left(\pi \eta_{1}+c\left(\eta_{2}-\epsilon \omega\right)\right), & C_{21}^{-}=-m \pi\left(m \pi \eta_{1}+c m\left(\eta_{2}-\epsilon \omega\right)\right), & C_{22}^{-}=-m \pi r \lambda\left(\eta_{2}-\epsilon \omega\right) .
\end{array}
$$

Let define a function $X(p)$ as:

$$
X(p)=\frac{C_{1} p^{3}+C_{2} p^{2}+C_{3} p+C_{4}}{\bar{C}_{1} p+\bar{C}_{2}}=0
$$

we obtain

$$
X(0)=\frac{\beta(\mathrm{cm} \epsilon+r \epsilon \lambda)(\delta+\beta \omega)\left(\mathcal{R}_{0}-1\right)}{\epsilon(\mathrm{cm}+\mathrm{r} \lambda)(\delta+\beta \omega)\left(\mathcal{R}_{0}-1\right)+\epsilon \delta(\mathrm{cm}+\mathrm{r} \lambda)^{\prime}}, \quad \lim _{p \rightarrow\left(\frac{\mathrm{m}}{\rho}\right)^{-}} X(p)=-\frac{\mathrm{mr} \epsilon \lambda}{\rho^{2}}<0 .
$$


Therefore, if $\mathcal{R}_{0}>1$ then $X(0)>0$ and there exists $p_{1} \in\left(0, \frac{m}{\rho}\right)$ such that $X\left(p_{1}\right)=0$. It follows from Eqs. (1.6)-(1.8) that

$$
\begin{aligned}
& x_{1}=\frac{\lambda}{m-\rho p_{1}}>0, \quad y_{1}=\frac{p_{1}\left(c+r x_{1}\right)}{\pi}>0, \\
& s_{1}=\frac{-\delta-p_{1} \eta_{1}-y_{1} \eta_{2}+\beta \omega+\sqrt{4 \beta \delta \omega+\left(-\delta-p_{1} \eta_{1}-y_{1} \eta_{2}+\beta \omega\right)^{2}}}{2 \delta \omega}>0 .
\end{aligned}
$$

Therefore, if $\mathcal{R}_{0}>1$, then the system has an infected equilibrium $E_{1}=\left(s_{1}, y_{1}, p_{1}, x_{1}\right)$. Now we show that $\mathrm{E}_{0} \in \Gamma$ and $\mathrm{E}_{1} \in \stackrel{\circ}{\Gamma}$. Clearly, $\mathrm{E}_{0} \in \Gamma$. From the equilibria conditions of $\mathrm{E}_{1}$ we have

$$
\beta=\delta s_{1}+\frac{\eta_{1} s_{1} p_{1}}{1+\omega s_{1}}+\frac{\eta_{2} s_{1} y_{1}}{1+\omega s_{1}} \Rightarrow \delta s_{1}+\epsilon y_{1}=\beta \Rightarrow 0<s_{1}<\frac{\beta}{\delta} \leqslant M_{1}, 0<y_{1}<\frac{\beta}{\epsilon} \leqslant M_{1} .
$$

Moreover, from Eqs. (1.7) and (1.8) we have

$$
\begin{gathered}
\mathrm{cp}_{1}=\pi \mathrm{y}_{1}+\frac{\mathrm{r}}{\rho} \lambda-\frac{\mathrm{mr}}{\rho} x_{1} \Rightarrow \mathrm{cp}_{1}+\frac{\mathrm{mr}}{\rho} x_{1}=\pi \mathrm{y}_{1}+\frac{\mathrm{r}}{\rho} \lambda<\pi \mathrm{M}_{1}+\frac{\mathrm{r}}{\rho} \lambda, \\
\mathrm{p}_{1}<\frac{\pi \mathrm{M}_{1}+\frac{\mathrm{r}}{\rho} \lambda}{\mathrm{c}} \leqslant \mathrm{M}_{2}, \quad x_{1}<\frac{\rho}{\mathrm{r}} \frac{\pi \mathrm{M}_{1}+\frac{\mathrm{r}}{\rho} \lambda}{\mathrm{m}} \leqslant \frac{\rho \mathrm{M}_{2}}{\mathrm{r}}=\mathrm{M}_{3} .
\end{gathered}
$$

It follows that, $\mathrm{E}_{1} \in \stackrel{\circ}{\Gamma}$.

\section{Global properties}

To investigate the global stability of the equilibria we construct Lyapunov functions using the method presented [30] and followed by [11, 12, 15-24, 26, 28, 29, 42]. Define $F(v)=v-1-\ln v$.

Theorem 2.1. For system (1.1)-(1.4), if $\mathcal{R}_{0} \leqslant 1$, then $\mathrm{E}_{0}$ is globally asymptotically stable in $\Gamma$.

Proof. Let $\mathcal{R}_{0} \leqslant 1$ and construct a Lyapunov function $\mathrm{U}_{0}(s, y, p, x)$ as:

$$
\mathrm{U}_{0}(s, y, p, x)=s-s_{0}-\int_{s_{0}}^{s} \frac{s_{0}(1+\omega \theta)}{\theta\left(1+\omega s_{0}\right)} d \theta+y+\frac{\eta_{1} s_{0}}{\left(c+r x_{0}\right)\left(1+\omega s_{0}\right)} p+\frac{r \eta_{1} s_{0}}{\rho\left(c+r x_{0}\right)\left(1+\omega s_{0}\right)} x_{0} F\left(\frac{x}{x_{0}}\right) .
$$

Clearly, $\mathrm{U}_{0}(\mathrm{~s}, \mathrm{y}, \mathrm{p}, \mathrm{x})>0$ for all $s, y, p, x>0$ and $\mathrm{U}_{0}\left(\mathrm{~s}_{0}, 0,0, \mathrm{x}_{0}\right)=0$. Calculating $\frac{\mathrm{du}_{0}}{\mathrm{dt}}$ along system (1.1)(1.4) we obtain

$$
\begin{aligned}
\frac{d U_{0}}{d t}= & \left(1-\frac{s_{0}(1+\omega s)}{s\left(1+\omega s_{0}\right)}\right)\left(\beta-\delta s-\frac{\eta_{1} s p}{1+\omega s}-\frac{\eta_{2} s y}{1+\omega s}\right)+\frac{\eta_{1} s p}{1+\omega s}+\frac{\eta_{2} s y}{1+\omega s}-\epsilon y \\
& +\frac{\eta_{1} s_{0}}{\left(c+r x_{0}\right)\left(1+\omega s_{0}\right)}(\pi y-c p-r x p)+\frac{r \eta_{1} s_{0}}{\rho\left(c+r x_{0}\right)\left(1+\omega s_{0}\right)}\left(1-\frac{x_{0}}{x}\right)(\lambda+\rho x p-m x) \\
= & \left(1-\frac{s_{0}(1+\omega s)}{s\left(1+\omega s_{0}\right)}\right)(\beta-\delta s)+\frac{\eta_{2} s_{0} y}{1+\omega s_{0}}-\epsilon y+\frac{\eta_{1} s_{0}}{\left(c+r x_{0}\right)\left(1+\omega s_{0}\right)} \pi y \\
& +\frac{r \eta_{1} s_{0}}{\rho\left(c+r x_{0}\right)\left(1+\omega s_{0}\right)}\left(1-\frac{x_{0}}{x}\right)(\lambda-m x) .
\end{aligned}
$$

Substituting $\beta=\delta s_{0}$ and $\lambda=m x_{0}$ we get

$$
\begin{aligned}
\frac{d U_{0}}{d t} & =-\delta \frac{\left(s-s_{0}\right)^{2}}{s\left(1+\omega s_{0}\right)}+\epsilon\left(\frac{\eta_{2} s_{0}}{\epsilon\left(1+\omega s_{0}\right)}+\frac{\eta_{1} s_{0} \pi}{\epsilon\left(c+r x_{0}\right)\left(1+\omega s_{0}\right)}-1\right) y-\frac{r \eta_{1} s_{0} m}{\rho\left(c+r x_{0}\right)\left(1+\omega s_{0}\right)} \frac{\left(x-x_{0}\right)^{2}}{x} \\
& =-\delta \frac{\left(s-s_{0}\right)^{2}}{s\left(1+\omega s_{0}\right)}-\frac{r \eta_{1} s_{0} m}{\rho\left(c+r x_{0}\right)\left(1+\omega s_{0}\right)} \frac{\left(x-x_{0}\right)^{2}}{x}+\epsilon\left(\mathcal{R}_{0}-1\right) y .
\end{aligned}
$$

If $\mathcal{R}_{0} \leqslant 1$, then $\frac{\mathrm{du}_{0}}{\mathrm{dt}} \leqslant 0$ for all $s, y, p, x>0$ and $\frac{\mathrm{du}_{0}}{\mathrm{dt}}=0$ when $s=s_{0}, x=x_{0}$ and $y=0$. It can be easily shown that $\frac{\mathrm{du}_{0}}{\mathrm{dt}}=0$ at $\mathrm{E}_{0}$. Applying LaSalle's invariance principle, we get $E_{0}$ is globally asymptotically stable when $\mathcal{R}_{0} \leqslant 1$. 
Theorem 2.2. For system (1.1)-(1.4), if $\mathcal{R}_{0}>1$, then $\mathrm{E}_{1}$ is globally asymptotically stable in $\stackrel{\circ}{\Gamma}$.

Proof. Let a function $\mathrm{U}_{1}(s, y, p, x)$ be defined as:

$\mathrm{U}_{1}(s, y, p, x)=s-s_{1}-\int_{s_{1}}^{s} \frac{s_{1}(1+\omega \theta)}{\theta\left(1+\omega s_{1}\right)} d \theta+y_{1} F\left(\frac{y}{y_{1}}\right)+\frac{\eta_{1} s_{1} p_{1}}{\pi y_{1}\left(1+\omega s_{1}\right)} p_{1} F\left(\frac{p}{p_{1}}\right)+\frac{\eta_{1} s_{1} p_{1}}{\rho \pi y_{1}\left(1+\omega s_{1}\right)} x_{1} F\left(\frac{x}{x_{1}}\right)$.

Clearly, $u_{1}(s, y, p, x)>0$ for all $s, y, p, x>0$ and $u_{1}\left(s_{1}, y_{1}, p_{1}, x_{1}\right)=0$. Calculating $\frac{d u_{1}}{d t}$ along the trajectories of (1.1)-(1.4) we obtain

$$
\begin{aligned}
\frac{d u_{1}}{d t}= & \left(1-\frac{s_{1}(1+\omega s)}{s\left(1+\omega s_{1}\right)}\right)\left(\beta-\delta s-\frac{\eta_{1} s p}{1+\omega s}-\frac{\eta_{2} s y}{1+\omega s}\right)+\left(1-\frac{y_{1}}{y}\right)\left(\frac{\eta_{1} s p}{1+\omega s}+\frac{\eta_{2} s y}{1+\omega s}-\epsilon y\right) \\
& +\frac{\eta_{1} s_{1} p_{1}}{\pi y_{1}\left(1+\omega s_{1}\right)}\left(1-\frac{p_{1}}{p}\right)(\pi y-c p-r x p)+\frac{\eta_{1} s_{1} p_{1}}{\rho \pi y_{1}\left(1+\omega s_{1}\right)}\left(1-\frac{x_{1}}{x}\right)(\lambda+\rho x p-m x) \\
= & \left(1-\frac{s_{1}(1+\omega s)}{s\left(1+\omega s_{1}\right)}\right)(\beta-\delta s)+\frac{\eta_{1} s_{1} p}{1+\omega s_{1}}+\frac{\eta_{2} s_{1} y}{1+\omega s_{1}}-\frac{\eta_{1} s p}{1+\omega s} \frac{y_{1}}{y}-\frac{\eta_{2} s y}{1+\omega s} \frac{y_{1}}{y}-\epsilon y+\epsilon y_{1} \\
& +\frac{\eta_{1} s_{1} p_{1}}{1+\omega s_{1}} \frac{y}{y_{1}}-\frac{\eta_{1} s_{1} p_{1}}{1+\omega s_{1}} \frac{p_{1} y}{p y_{1}}-\frac{\eta_{1} s_{1} p_{1}}{\pi y_{1}\left(1+\omega s_{1}\right)} c p+\frac{\eta_{1} s_{1} p_{1}}{\pi y_{1}\left(1+\omega s_{1}\right)} c p_{1}+\frac{\eta_{1} s_{1} p_{1}}{\pi y_{1}\left(1+\omega s_{1}\right)} r x p_{1} \\
& -\frac{r \eta_{1} s_{1} p_{1}}{\pi y_{1}\left(1+\omega s_{1}\right)} x_{1} p+\frac{\eta_{1} s_{1} p_{1}}{\rho \pi y_{1}\left(1+\omega s_{1}\right)}\left(1-\frac{x_{1}}{x}\right)(\lambda-m x) .
\end{aligned}
$$

Applying the equilibrium conditions for $E_{1}$

$$
\beta=\delta s_{1}+\frac{\eta_{1} s_{1} p_{1}}{1+\omega s_{1}}+\frac{\eta_{2} s_{1} y_{1}}{1+\omega s_{1}}, \quad \epsilon y_{1}=\frac{\eta_{1} s_{1} p_{1}}{1+\omega s_{1}}+\frac{\eta_{2} s_{1} y_{1}}{1+\omega s_{1}}, \quad c p_{1}=\pi y_{1}-r x_{1} p_{1}, \quad \lambda=m x_{1}-\rho x_{1} p_{1} .
$$

we get

$$
\begin{aligned}
\frac{d u_{1}}{d t}= & -\delta \frac{\left(s-s_{1}\right)^{2}}{s\left(1+\omega s_{1}\right)}+\left(1-\frac{s_{1}(1+\omega s)}{s\left(1+\omega s_{1}\right)}\right)\left(\frac{\eta_{1} s_{1} p_{1}}{1+\omega s_{1}}+\frac{\eta_{2} s_{1} y_{1}}{1+\omega s_{1}}\right) \\
& -\frac{\eta_{1} s_{1} p_{1}}{1+\omega s_{1}} \frac{s p y_{1}\left(1+\omega s_{1}\right)}{s_{1} p_{1} y(1+\omega s)}-\frac{\eta_{2} s_{1} y_{1}}{1+\omega s_{1}} \frac{s\left(1+\omega s_{1}\right)}{s_{1}(1+\omega s)}+\frac{\eta_{1} s_{1} p_{1}}{1+\omega s_{1}}+\frac{\eta_{2} s_{1} y_{1}}{1+\omega s_{1}} \\
& -\frac{\eta_{1} s_{1} p_{1}}{1+\omega s_{1}} \frac{p_{1} y}{p y_{1}}+\frac{\eta_{1} s_{1} p_{1}}{1+\omega s_{1}}-2 \frac{\eta_{1} s_{1} p_{1}}{\pi y_{1}\left(1+\omega s_{1}\right)} r x_{1} p_{1}+\frac{\eta_{1} s_{1} p_{1}}{\pi y_{1}\left(1+\omega s_{1}\right)} r x p_{1} \\
& +\frac{\eta_{1} s_{1} p_{1}}{\pi y_{1}\left(1+\omega s_{1}\right)} r x_{1} p_{1} \frac{x_{1}}{x}-\frac{r \eta_{1} s_{1} p_{1} m}{\rho \pi y_{1}\left(1+\omega s_{1}\right)} \frac{\left(x-x_{1}\right)^{2}}{x} .
\end{aligned}
$$

Eq. (2.1) can be simplified as:

$$
\begin{aligned}
\frac{d u_{1}}{d t}= & -\delta \frac{\left(s-s_{1}\right)^{2}}{s\left(1+\omega s_{1}\right)}+\frac{\eta_{1} s_{1} p_{1}}{1+\omega s_{1}}\left[3-\frac{s_{1}(1+\omega s)}{s\left(1+\omega s_{1}\right)}-\frac{s p y_{1}\left(1+\omega s_{1}\right)}{s_{1} p_{1} y(1+\omega s)}-\frac{p_{1} y}{p y_{1}}\right] \\
& +\frac{\eta_{2} s_{1} y_{1}}{1+\omega s_{1}}\left[2-\frac{s_{1}(1+\omega s)}{s\left(1+\omega s_{1}\right)}-\frac{s\left(1+\omega s_{1}\right)}{s_{1}(1+\omega s)}\right]-\frac{\eta_{1} s_{1} p_{1}}{\pi y_{1}\left(1+\omega s_{1}\right)} r x_{1} p_{1}\left[2-\frac{x}{x_{1}}-\frac{x_{1}}{x}\right] \\
& -\frac{\eta_{1} s_{1} p_{1} m}{\rho \pi y_{1}\left(1+\omega s_{1}\right)} \frac{\left(x-x_{1}\right)^{2}}{x} \\
= & -\delta \frac{\left(s-s_{1}\right)^{2}}{s\left(1+\omega s_{1}\right)}-\frac{\eta_{1} s_{1} p_{1}}{\pi y_{1}\left(1+\omega s_{1}\right)} \frac{r \lambda}{\rho x_{1}} \frac{\left(x-x_{1}\right)^{2}}{x}+\frac{\eta_{1} s_{1} p_{1}}{1+\omega s_{1}}\left[3-\frac{s_{1}(1+\omega s)}{s\left(1+\omega s_{1}\right)}-\frac{s p y_{1}\left(1+\omega s_{1}\right)}{s_{1} p_{1} y(1+\omega s)}-\frac{p_{1} y}{p y_{1}}\right] \\
& +\frac{\eta_{2} s_{1} y_{1}}{1+\omega s_{1}}\left[2-\frac{s_{1}(1+\omega s)}{s\left(1+\omega s_{1}\right)}-\frac{s\left(1+\omega s_{1}\right)}{s_{1}(1+\omega s)}\right] .
\end{aligned}
$$

Using the rule

$$
\frac{1}{n} \sum_{i=1}^{n} a_{i} \geqslant \sqrt[n]{\prod_{i=1}^{n} a_{i}}, \quad \text { where, } \quad a_{i} \geqslant 0, i=1,2, \ldots, n
$$

we get

$$
\frac{1}{3}\left(\frac{s_{1}(1+\omega s)}{s\left(1+\omega s_{1}\right)}+\frac{s p y_{1}\left(1+\omega s_{1}\right)}{s_{1} p_{1} y(1+\omega s)}+\frac{p_{1} y}{p y_{1}}\right) \geqslant 1, \quad \frac{1}{2}\left(\frac{s_{1}(1+\omega s)}{s\left(1+\omega s_{1}\right)}+\frac{s\left(1+\omega s_{1}\right)}{s_{1}(1+\omega s)}\right) \geqslant 1 .
$$


Therefore, $\frac{\mathrm{du}_{1}}{\mathrm{dt}} \leqslant 0$ for all $s, y, p, x>0$ and $\frac{\mathrm{du}_{1}}{\mathrm{dt}}=0$ if and only if $s=s_{1}, y=y_{1}, p=p_{1}$ and $x=x_{1}$. It follows that the global stability of $E_{1}$ is induced from LaSalle's invariance principle.

\section{Numerical simulations}

Using the values in Table 1, we consider two cases as follows:

Case 1: We simulate system (1.1)-(1.4) with the following initial conditions:

IC1: $s(0)=14.0, y(0)=1.0, p(0)=1.5$, and $x(0)=1.5$;

IC2: $s(0)=8.0, y(0)=2.0, p(0)=3.0$, and $x(0)=4.0$;

IC3: $s(0)=4.0, y(0)=3.5, p(0)=6.0$, and $x(0)=7.0$.

We fix $\omega=0.09$ and consider the values of $\eta_{1}$ and $\eta_{2}$ as following sets:

Set (I): We let $\eta_{1}=\eta_{2}=0.001$. Computing $\mathcal{R}_{0}=0.0857<1$, Figures (1)-(4) show that, $E_{0}=\left(s_{0}, 0,0, x_{0}\right)$ is globally asymptotically stable, where $s_{0}=\frac{\beta}{\delta}=20$ and $x_{0}=\frac{\lambda}{m}=1.4$, which agrees with the result of (2.1).

Set (II): We choose $\eta_{1}=\eta_{2}=0.05$. Calculating $\mathcal{R}_{0}=4.2857>1$, we compute the equilibria as $\mathrm{E}_{0}(20.0,0,0,1.4)$ and $\mathrm{E}_{1}=(6.66,2.66,3.73,5.51)$. We have observed that in Figures (1)-(4), when $\mathcal{R}_{0}>1$, the solution of the system tend to $E_{1}$ for IC1-IC3 and (2.2) is confirmed.

Case 2. We fixed the value $\eta_{1}=\eta_{2}=0.06$, by using the following initial conditions $s(0)=7, y(0)=$ $2.0, p(0)=3.0$, and $x(0)=4.0$, we can see from Figures (5)-(8) that the evolution of the system's states with different values of $\omega$. We have observed that $\mathcal{R}_{0}>1$, and the trajectory of the system converges to the equilibrium $E_{1}$ for smaller values of $\omega$ e.g. $\omega=0.0,0.2,0.4$. Whereas, $\mathcal{R}_{0} \leqslant 1$, and the system has one equilibrium $E_{0}$ when $\omega$ become larger e.g. $\omega=2,5$. Let $\omega^{\text {ct }}$ be the critical value of the parameter $\omega$, such that

$$
\mathcal{R}_{0}=\frac{\left(\eta_{1} \pi m+\eta_{2} c m+\eta_{2} r \lambda\right) \beta}{\epsilon(c m+r \lambda)\left(\delta+\beta \omega^{c t}\right)}=1
$$

Using the data given in Table 1 , we obtain $\omega^{\text {ct }}=0.67$. The variation of $\mathcal{R}_{0}$ w.r.t. $\omega$ are listed in Table 2 . We can observed that as $\omega$ is increased then $\mathcal{R}_{0}$ is decreased. Moreover, we have the following cases:

(i) if $0 \leqslant \omega<0.67$, then $E_{1}$ exists and it is globally asymptotically stable,

(ii) if $\omega \geqslant 0.67$, then $E_{0}$ is globally asymptotically stable.

Table 1: The value of the parameters of model (1.1)-(1.4).

\begin{tabular}{|c|c|c|c|}
\hline Parameter & Value & Parameter & Value \\
\hline$\beta$ & 2 & $\delta$ & 0.1 \\
\hline$\eta_{1}$ & varied & $\eta_{2}$ & varied \\
\hline$\pi$ & 4 & $\mathrm{c}$ & 0.1 \\
\hline $\mathrm{r}$ & 0.5 & $\lambda$ & 1.4 \\
\hline $\mathrm{m}$ & 1 & $\rho$ & 0.2 \\
\hline$\omega$ & varied & $\epsilon$ & 0.5 \\
\hline
\end{tabular}

Table 2: The value of $\mathcal{R}_{0}$ for different values of $\omega$.

\begin{tabular}{|c|c|c|}
\hline$\omega$ & Equilibria & $\mathcal{R}_{0}$ \\
\hline 0.0 & $(3.77,3.24,3.91,6.43)$ & 14.3997 \\
\hline 0.2 & $(8.93,2.21,3.54,4.80)$ & 2.8800 \\
\hline 0.4 & $(15.93,0.08,2.25,2.60)$ & 1.6000 \\
\hline 0.67 & $(20.00,0.00,0.00,1.40)$ & 1.00 \\
\hline 1 & $(20.00,0.00,0.00,1.40)$ & 0.6857 \\
\hline 5 & $(20.00,0.00,0.00,1.40)$ & 0.1426 \\
\hline
\end{tabular}




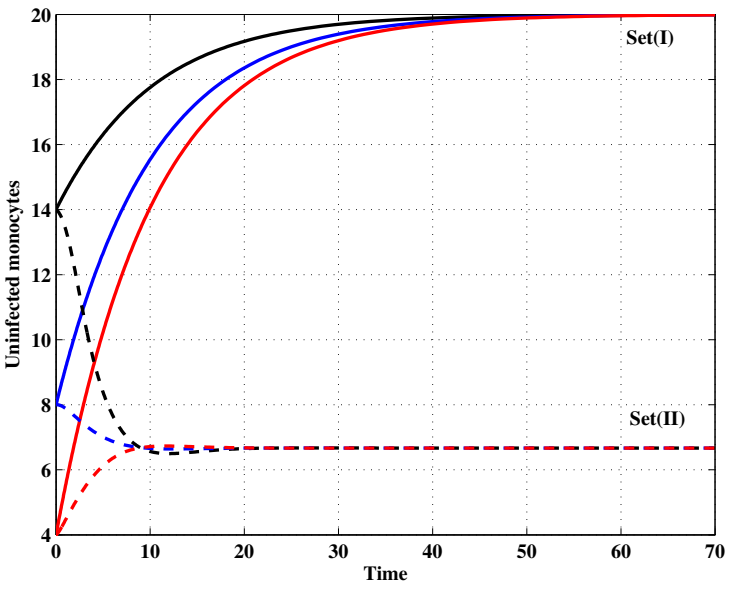

Figure 1: Uninfected monocytes.

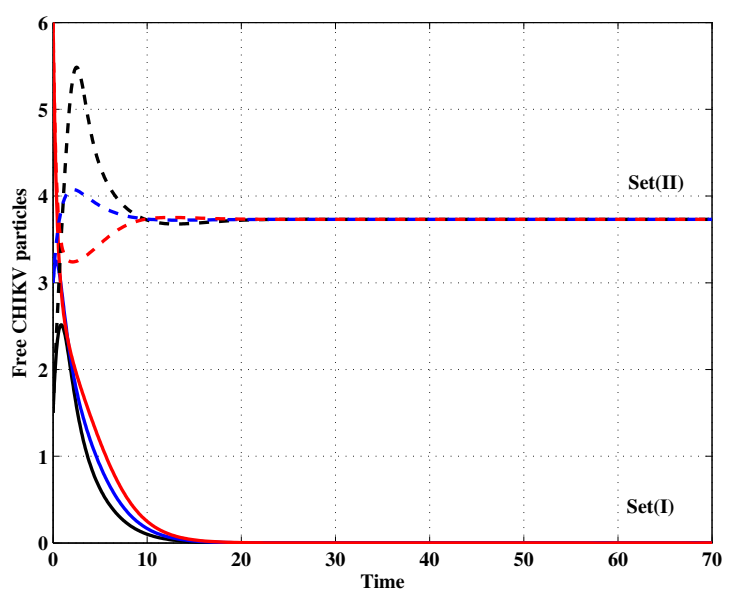

Figure 3: Free CHIKV particles.

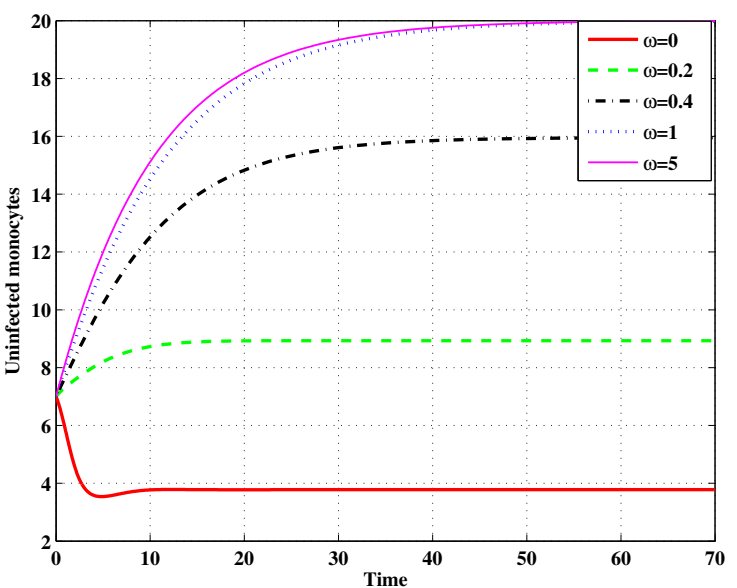

Figure 5: Uninfected monocytes.

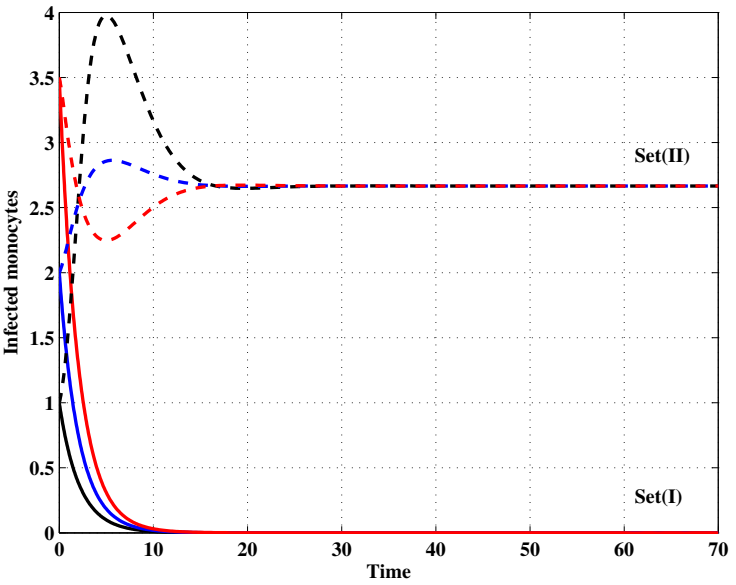

Figure 2: Infected monocytes.

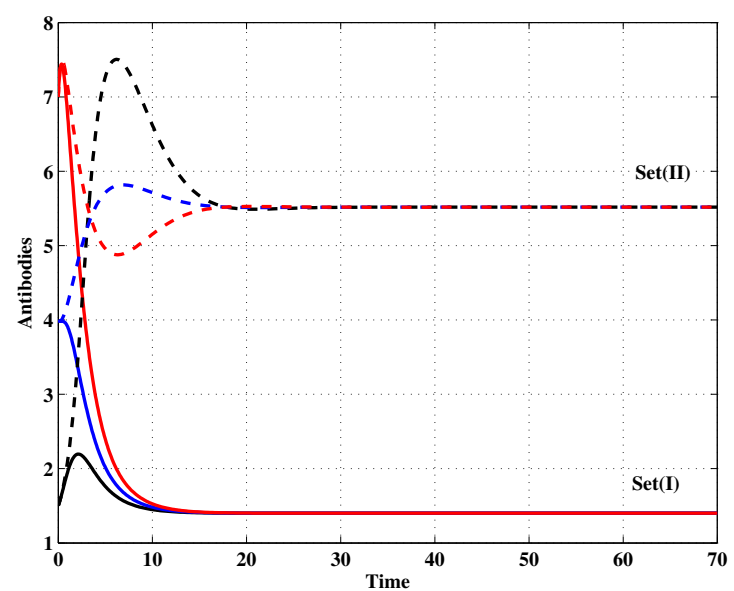

Figure 4: Antibodies.

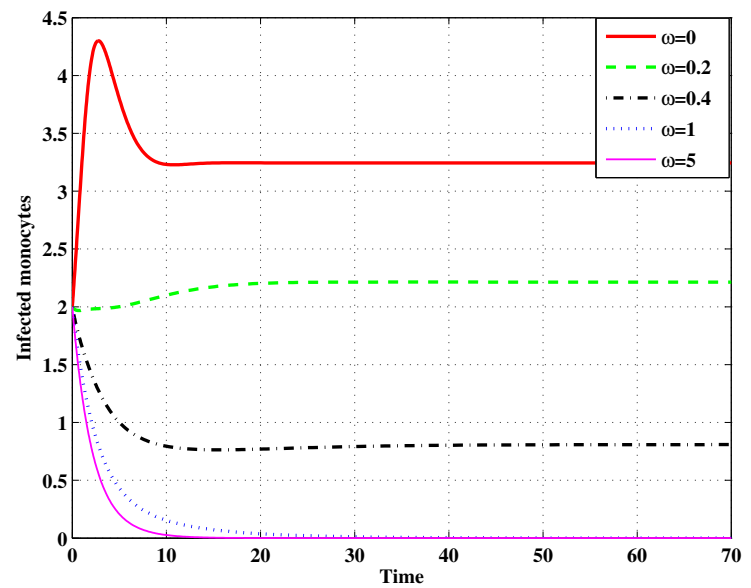

Figure 6: Infected monocytes. 


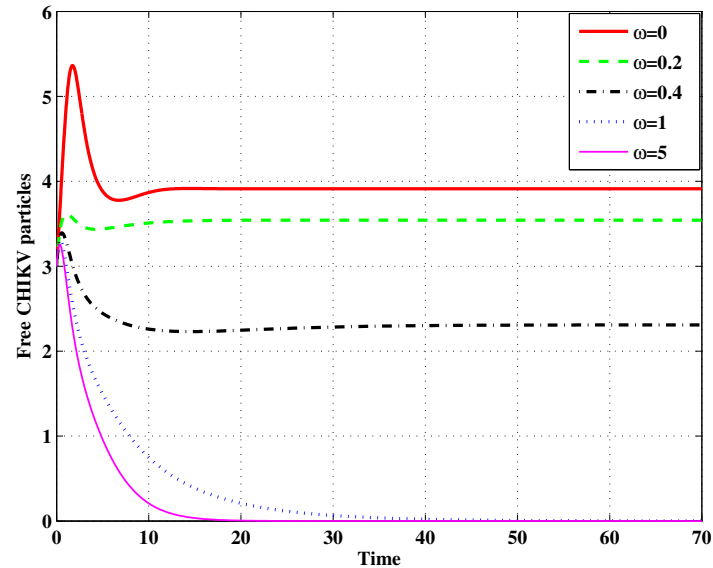

Figure 7: Free CHIKV particles.

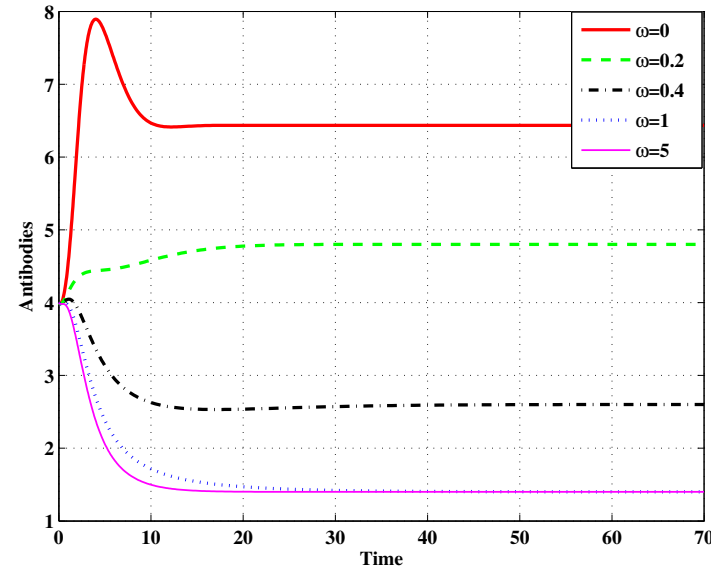

Figure 8: Antibodies.

\section{References}

[1] A. Abdelrazec, J. Belair, C. H. Shan, H. P. Zhu, Modeling the spread and control of dengue with limited public health resources, Math. Biosci., 271 (2016), 136-145. 1

[2] F. B. Agusto, S. Bewick, W. F. Fagan, Mathematical model of Zika virus with vertical transmission, Infectious Disease Modelling, 2 (2017), 244-267. 1

[3] E. Beretta, V. Capasso, D. G. Garao, A mathematical model for malaria transmission with asymptomatic carriers and two age groups in the human population, Math. Biosci., 300 (2018), 87-101. 1

[4] E. Bonyah, K. O. Okosun, Mathematical modeling of Zika virus, Asian Pacific J. Tropical Disease, 6 (2016), 673-679. 1

[5] N. Chitnis, J. M. Cushing, J. M. Hyman, Bifurcation analysis of a mathematical model for malaria transmission, SIAM J. Appl. Math., 67 (2006), 24-45. 1

[6] N. Chitnis, J. M. Hyman, J. M. Cushing, Determining important parameters in the spread of malaria through the sensitivity analysis of a mathematical model, Bull. Math. Biol., 70 (2008), 1272-1296. 1

[7] E. Dantas, M. Tosin, A. Cunha, Calibration of a SEIR-SEI epidemic model to describe the Zika virus outbreak in Brazil, Appl. Math. Comput., 338 (2018), 249-259. 1

[8] Y. Dumont, F. Chiroleu, Vector control for the chikungunya disease, Math. Biosci. Eng., 7 (2010), 313-345. 1

[9] Y. Dumont, F. Chiroleu, C. Domerg, On a temporal model for the chikungunya disease: modeling, theory and numerics, Math. Biosci., 213 (2008), 80-91.

[10] Y. Dumont, J. M. Tchuenche, Mathematical studies on the sterile insect technique for the chikungunya disease and aedes albopictus, J. Math. Biol., 65 (2012), 809-854. 1

[11] A. M. Elaiw, Global properties of a class of HIV models, Nonlinear Anal. Real World Appl., 11 (2010), 2253-2263. 2

[12] A. M. Elaiw, Global properties of a class of virus infection models with multitarget cells, Nonlinear Dynam., 69 (2012), 423-435. 2

[13] A. M. Elaiw, T. O. Alade, S. M. Alsulami, Analysis of latent CHIKV dynamics models with general incidence rate and time delays, J. Biol. Dyn., 12 (2018), 700-730. 1

[14] A. M. Elaiw, T. O. Alade, S. M. Alsulami, Analysis of within-host CHIKV dynamics models with general incidence rate, Int. J. Biomath., 11 (2018), 25 pages. 1

[15] A. M. Elaiw, S. E. Almalki, A. D. Hobiny, Stability of CHIKV infection models with CHIKV-monocyte and infectedmonocyte saturated incidences, AIP Advances, 9 (2019), 12 pages. 1, 2

[16] A. M. Elaiw, N. A. Almuallem, Global properties of delayed-HIV dynamics models with differential drug efficacy in cocirculating target cells, Appl. Math. Comput., 265 (2015), 1067-1089.

[17] A. M. Elaiw, N. A. Almuallem, Global dynamics of delay-distributed HIV infection models with differential drug efficacy in cocirculating target cells, Math. Methods Appl. Sci., 39 (2016), 4-31.

[18] A. M. Elaiw, N. H. AlShamrani, Global stability of humoral immunity virus dynamics models with nonlinear infection rate and removal, Nonlinear Anal. Real World Appl., 26 (2015), 161-190.

[19] A. M. Elaiw, N. H. AlShamrani, Stability of a general delay-distributed virus dynamics model with multi-staged infected progression and immune response, Math. Methods Appl. Sci., 40 (2017), 699-719.

[20] A. M. Elaiw, N. H. AlShamrani, Stability of an adaptive immunity pathogen dynamics model with latency and multiple delays, Math. Methods Appl. Sci., 41 (2018), 6645-6672.

[21] A. M. Elaiw, S. A. Azoz, Global properties of a class of HIV infection models with Beddington-DeAngelis functional response, Math. Methods Appl. Sci., 36 (2013), 383-394. 
[22] A. M. Elaiw, E. K. Elnahary, A. A. Raezah, Effect of cellular reservoirs and delays on the global dynamics of HIV, Adv. Difference Equ., 2018 (2018), 36 pages.

[23] A. M. Elaiw, I. Hassanien, S. A. Azoz, Global stability of HIV infection models with intracellular delays, J. Korean Math. Soc., 49 (2012), 779-794.

[24] A. M. Elaiw, A. A. Raezah, Stability of general virus dynamics models with both cellular and viral infections and delays, Math. Methods Appl. Sci., 40 (2017), 5863-5880. 1, 2

[25] A. M. Elaiw, A. A. Raezah, B. S. Alofi, Dynamics of delayed pathogen infection models with pathogenic and cellular infections and immune impairment, AIP Advances, 8 (2018), 14 pages. 1

[26] A. M. Elaiw, A. A. Raezah, S. A. Azoz, Stability of delayed HIV dynamics models with two latent reservoirs and immune impairment, Adv. Difference Equ., 2018 (2018), 25 pages. 2

[27] L. Esteva, C. Vargas, A model for dengue disease with variable human population, J. Math. Biol., 38 (1999), 220-240. 1

[28] A. D. Hobiny, A. M. Elaiw, A. A. Almatrafi, Stability of delayed pathogen dynamics models with latency and two routes of infection, Adv. Difference Equ., 2018 (2018), 26 pages. 2

[29] G. Huang, Y. Takeuchi, W. Ma, Lyapunov functionals for delay differential equations model of viral infections, SIAM J. Appl. Math., 70 (2010), 2693-2708. 2

[30] A. Korobeinikov, Global properties of basic virus dynamics models, Bull. Math. Biol., 66 (2004), 879-883. 2

[31] X. L. Lai, X. F. Zou, Modelling HIV-1 virus dynamics with both virus-to-cell infection and cell-to-cell transmission, SIAM J. Appl. Math., 74 (2014), 898-917. 1

[32] X. L. Lai, X. F. Zou, Modeling cell-to-cell spread of HIV-1 with logistic target cell growth, J. Math. Anal. Appl., 426 (2015), 563-584.

[33] F. Li, J. L. Wang, Analysis of an HIV infection model with logistic target cell growth and cell-to-cell transmission, Chaos Solitons Fractals, 81 (2015), 136-145. 1

[34] X. Z. Liu, P. Stechlinski, Application of control strategies to a seasonal model of chikungunya disease, Appl. Math. Model., 39 (2015), 3194-3220. 1

[35] K. M. Long, M. T. Heise, Protective and pathogenic responses to chikungunya virus infection, Curr. Trop. Med. Rep., 2 (2015), 13-21. 1

[36] S. Mandal, R. R. Sarkar, S. Sinha, Mathematical models of malaria-a review, Malaria J., 10 (2011), 19 pages. 1

[37] C. A. Manore, K. S. Hickmann, S. Xu, H. J. Wearing, J. M. Hyman, Comparing dengue and chikungunya emergence and endemic transmission in A. aegypti and A. albopictus, J. Theoret. Biol., 356 (2014), 174-191. 1

[38] D. Moulay, M. Aziz-Alaoui, M. Cadivel, The chikungunya disease: modeling, vector and transmission global dynamics, Math. Biosci., 229 (2011), 50-63.

[39] D. Moulay, M. Aziz-Alaoui, H.-D. Kwon, Optimal control of chikungunya disease: larvae reduction, treatment and prevention, Math. Biosci. Eng., 9 (2012), 369-392. 1

[40] S. M. Raimundo, M. Amaku, E. Massad, Equilibrium analysis of a yellow fever dynamical model with vaccination, Comput. Math. Methods Med., 2015 (2015), 12 pages. 1

[41] H. Y. Shu, Y. M. Chen, L. Wang, Impacts of the cell-free and cell-to-cell infection modes on viral dynamics, J. Dynam. Differential Equations, 30 (2018), 1817-1836. 1

[42] H. Y. Shu, L. Wang, J. Watmough, Global stability of a nonlinear viral infection model with infinitely distributed intracellular delays and CTL immune responses, SIAM J. Appl. Math., 73 (2013), 1280-1302. 2

[43] J. J. Tewaa, J. L. Dimi, S. Bowong, Lyapunov functions for a dengue disease transmission model, Chaos Solitons Fractals, 39 (2009), 936-941. 1

[44] J. L. Wang, J. Y. Lang, X. F. Zou, Analysis of an age structured HIV infection model with virus-to-cell infection and cell-to-cell transmission, Nonlinear Anal. Real World Appl., 34 (2017), 75-96. 1

[45] Y. Wang, X. N. Liu, Stability and Hopf bifurcation of a within-host chikungunya virus infection model with two delays, Math. Comput. Simulation, 138 (2017), 31-48. 1

[46] L. Yakob, A. C. Clements, A mathematical model of chikungunya dynamics and control: the major epidemic on Reunion Island, PLoS One, 8 (2013), 6 pages. 1

[47] Y. Yang, L. Zou, S. G. Ruan, Global dynamics of a delayed within-host viral infection model with both virus-to-cell and cell-to-cell transmissions, Math. Biosci., 270 (2015), 183-191. 1

[48] M. Zhu, Y. Xu, A time-periodic dengue fever model in a heterogeneous environment, Math. Comput. Simulation, 155 (2019), 115-129. 1 